provide assessment, operative intervention and postoperative care to a paediatric cohort.

Results Twelve operations were undertaken for ten patients (table 1) with a median age of 3.4 years (IQR [2.3-5.8]) and weight $11.9 \mathrm{~kg}$ (IQR [9.1-14.5]). For those requiring cardiopulmonary bypass (CPB), median time was $107 \mathrm{~min}$ (IQR [92150]) and cross-clamp (XC) time $73 \mathrm{~min}$ (IQR [49.2-98.5]).

\begin{tabular}{llllll}
\multicolumn{7}{l}{ Abstract G31(P) Table 1 } & & & \\
\hline No. & $\begin{array}{l}\text { Age } \\
\text { (Years) }\end{array}$ & Procedure & $\begin{array}{l}\text { CPB } \\
\text { (Mins) }\end{array}$ & $\begin{array}{l}\text { XC } \\
\text { (Mins) }\end{array}$ & $\begin{array}{l}\text { Extubation Time } \\
\text { (Hours) }\end{array}$ \\
\hline 1 & 2 & VSD Closure & 60 & 34 & 2 \\
2 & 4.25 & REV Procedure (PA-VSD) & 320 & 226 & 58.08 \\
3 & 6.91 & AVSD Repair & 92 & 72 & 2.08 \\
4 & 0.02 & TAPVD Repair & 95 & 50 & 18.33 \\
5 & 6.33 & TOF Repair & 150 & 100 & 0.75 \\
6 & 3.08 & TOF Repair & 154 & 98 & 15.58 \\
7 & 2 & Partial PDA Occlusion & 0 & 0 & 3.75 \\
8 & 18.67 & Redo PV Implantation & 111 & 0 & 4.5 \\
9 & 3 & VSD Closure, PA de-banding and & 107 & 75 & 1 \\
& & reconstruction & & & \\
10 & 3.75 & VSD and PFO Closure & 71 & 47 & 1.25 \\
\hline
\end{tabular}

Fast-track extubation was performed in $70 \%$ with no reintubations. Post-operative morbidity was low and only patient 2 returned to theatre for additional procedures: delayed sternal closure and subsequent re-exploration. Case 3 was complicated by Junctional Ectopic Tachycardia, which resolved with medical management.

The team performed the institution's first redo surgery and neonatal operation. The local cardiac surgeon led his first paediatric VSD closure and Tetralogy of Fallot repair. All patients were extubated and discharged, or eligible for ICU discharge prior to conclusion of the mission.

Visiting PICU staff were resident 24 hours a day. The local ICU team were supported by twice daily PICU rounds, multi-disciplinary meetings and bedside teaching for nurses and trainees. The visiting Cardiologist undertook outpatient consultations, intra-and post-operative echocardiography and ECHO teaching. A morning lecture series delivered education to local trainees on topics including cardiac output, inotropes, heart failure and the surgical management of congenital heart disease.

Conclusions Healing Little Hearts provides cardiac surgical and critical care in developing countries. Short, well-planned missions can deliver quality care to a high turnover of patients with safe, early extubation and minimal morbidity, despite limited resources. Future missions will provide ongoing training and support the development of a safe, independent program capable of handling carefully selected paediatric congenital cardiac surgeries.

\section{G32(P) LATE PRETERM INFANTS AT GREATER RISK OF PAEDIATRIC INTENSIVE CARE ADMISSION AND LONGER INVASIVE VENTILATION DUE TO RESPIRATORY SYNCITIAL VIRUS BRONCHIOLITIS}

S Followell, M Christopherson, P Davis. Paediatric Intensive Care, Bristol Royal Hospital for Children, Bristol, UK

10.1136/archdischild-2018-rcpch.31
Aims Only a small proportion of late-preterm (LPT) infants (34-36+6 weeks gestational age) require admission to NICU for respiratory support at birth. We aimed to evaluate the subsequent impact of Respiratory Syncitial Virus (RSV) on LPT infants compared to FT infants.

Methods A retrospective cohort study of infants admitted to a regional tertiary PICU with a primary diagnosis of RSV-positive bronchiolitis over a five-year period was undertaken. LPT infants were compared to FT infants (37 weeks and greater gestational age). The relative risk of PICU admission for RSVpositive bronchiolitis for LPT and FT infants in our regional population was calculated using Office of National Statistics data. Ventilation rates between the 2 groups admitted to PICU were compared using Chi-squared. Non-parametric statistical analysis was undertaken to identify any significant differences in other resource utilisation between the LPT and FT infants.

Results 40 LPT and 102 FT infants were admitted to PICU with a diagnosis of RSV-positive bronchiolitis from 2012 to 2017. Of infants born between 34 and 42 weeks in this period, 4.4\% were LPT. However, LPT infants constituted $28.2 \%$ of the study population admitted to PICU with RSVpositive bronchiolitis ( $\mathrm{RR}=8.44, \quad 95 \% \mathrm{CI}$ 5.85-12.15, $\mathrm{p}<0.0001)$. Invasive ventilation rates were similar between LPT and FT infants (75\% LPT vs. $68.6 \%$ FT, $\chi 2=0.08$, DF1, $\mathrm{p}=0.78$ ). The median length of invasive ventilation was 6 days (IQR 5-11) among LPT infants and 5 days (IQR 4-7) for FT infants $(\mathrm{W}=1322 ; \mathrm{p}=0.039)$. The median length of PICU stay was 7 days (IQR 5-12) for LPT infants and 6 days (IQR 4-8) among FT infants $(\mathrm{W}=2574 ; \mathrm{p}=0.015)$. There were no PICU deaths among the 142 RSV-positive bronchiolitis admissions.

Conclusion Despite few LPT infants requiring respiratory input from a neonatologist at birth, following discharge home, they are at significantly increased risk of admission to PICU with RSV-positive bronchiolitis, compared to FT infants, with longer periods of invasive ventilation and lengthier admissions. This may reflect the importance of reaching full term for lung development and the control of breathing, especially when exposed to common respiratory viruses during infancy.

\section{G33(P) OVERVIEW OF RARE RENAL DISEASES AT A PAEDIATRIC RENAL CENTRE THROUGH THE NATIONAL REGISTRY OF RARE KIDNEY DISEASES (RADAR) IN THE UNITED KINGDOM}

'L Kerecuk, 'M Kokocinska, 'S Parkes, ${ }^{1} \mathrm{~J}$ Kainth, ${ }^{2} \mathrm{M}$ Dillon. 'Nephrology, Birmingham Children's Hospital, Birmingham, UK; ${ }^{2}$ National Registry of Rare Kidney Diseases (RADAR), UK Renal Registry, Bristol, UK

\subsection{6/archdischild-2018-rcpch.32}

Aims Rare kidney diseases need better understanding and the starting point is collection of clinical data. Therefore our centre is participating in RaDaR which is a UK Renal Association initiative designed to gather information. RaDaR recruitment began in 2010 and now covers more than 40 conditions. There are around 10000 recruits from 78 renal adult and paediatric units in the UK. Our Paediatric Renal Centre is the leading recruiting hospital in the UK.

To describe the range of range of conditions and patient numbers recruited to RADAR at $\mathrm{BCH}$ which is a national tertiary renal referral hospital in the UK.

Methods The RaDaR dataset is defined by the UK Renal Registry in association with the Rare Disease Groups, made 
up of experts in each eligible condition. Data fields include demographics, blood and urine results, medications, transplant and dialysis history, genetics and co-morbidities. Data is entered retrospectively from the patient's medical records following consent.

Results 319 patients have been consented at $\mathrm{BCH}$. The age range is from birth to 16 years with mean of 4.9 years with male to female ratio of $55 \%: 45 \%$. The most common condition is Idiopathic Nephrotic Syndrome $(n=128 ; 39 \%)$, followed by Alport Syndrome $(n=33 ; 10 \%)$, ARPKD $(n=25$; $8 \%)$, Hyperoxaluria $(n=24 ; 7 \%)$ and STEC HUS $(n=22 ; 7 \%)$. The other conditions with numbers of patients recruited so far include: ADPKD $(n=14)$, aHUS $(n=13)$; Cystinosis $(n=9)$; Cystinuria $(n=3)$; Dent and Lowe $(n=7)$; HNF1b $(n=6)$; Hypokalaemic Alkalosis $(n=8)$; MPGN $(n=14)$ and Vasculitis $(n=7)$.

Conclusion $\mathrm{RaDaR}$ provides important epidemiology data based on the whole country population which is shared amongst the renal team to develop further research into rare kidney diseases and improve the quality of care for these patients. It also gives an opportunity to define the best treatment practices across the country in the future.

\section{G34(P) ABSTRACT WITHDRAWN}

\section{G35(P) ROLE OF LUMBAR PUNCTURE IN INFANTS PRESENTING WITH RECURRENT APNOEA}

R Manuel, P Ramesh. Paediatric Intensive Care Unit, University Hospital of North Midlands NHS Trust, Stoke on Trent, UK

\subsection{6/archdischild-2018-rcpch.33}

Background The commonest cause of recurrent apnoea in early infancy is due to bronchiolitis especially when they present with other respiratory symptoms. Lumbar puncture is commonly performed in these babies to rule out the possibility of meningitis. Almost all these babies are also commenced on antibiotics and antiviral agents to cover meningitis until it is excluded.

Aim To identify the incidence of meningitis in infants presenting with recurrent apnoea to a tertiary paediatric intensive care unit.

Methods Prospective collection of data and retrospective review of case notes of all the infants intubated and ventilated because of recurrent apnoea. The study period was for three and a half years from October 2014 to February 2017.

Results Sixty five infants were included in the study. Their mean age was 5 weeks (range: 3 weeks to 3 months). All of them had a non-bronchoscopic Broncho Alveolar Lavage (BAL) to identify respiratory pathogens and also had lumbar puncture to rule out meningitis. All the infants presented with a history of coryzal symptoms, poor feeding, respiratory distress and recurrent apnoea. The average highest peak CRP for this group was 73 (interquartile range: 26-103). 39 babies $(60 \%)$ received fluid resuscitation at presentation.

PCR test was positive in the BAL for a viral pathogen in sixty infants (92\%) and negative in five (8\%). Two babies in the
BAL positive group also had a positive PCR test for a virus in the CSF. One baby had enterovirus positive in both BAL and CSF and another baby was PCR positive for Rhinovirus in BAL and HHV6 in CSF. The CSF cell count was normal in both these infants. No infant had evidence of bacterial meningitis or a treatable cause of viral meningitis.

Conclusion Our results show that there is a low incidence of possible viral meningitis in infants presenting with recurrent apnoea and other respiratory symptoms. None of the babies would have benefitted from antibiotics or antiviral agents.

\section{G36(P) OUTCOMES OF INFANTS TRANSFERRED FROM THE NEONATAL INTENSIVE CARE TO THE PAEDIATRIC WARD AND PAEDIATRIC INTENSIVE CARE AFTER 44 WEEKS CORRECTED GESTATIONAL AGE}

\begin{abstract}
${ }^{1,2} \mathrm{~K}$ Crawford, 'SZ Kamupira, 'S Morley, ${ }^{2} \mathrm{AW}$ Kelsall. ${ }^{1}$ Paediatric Intensive Care, Cambridge University NHS Foundation Trust, Cambridge, UK; ${ }^{2}$ Neonatology, Cambridge University NHS Foundation Trust, Cambridge, UK
\end{abstract}

10.1136/archdischild-2018-rcpch.34

Aim NHS England has outlined the Neonatal Intensive Care Unit (NICU) service to be for infants who are generally (but not exclusively) $<44$ weeks corrected gestational age (CGA). There are implications for the Paediatric Intensive Care Unit (PICU) and general paediatric wards in terms of managing this workload, and transitioning complex infants. Following challenges in accommodating some of these cases within our services, we conducted a study to assess the workload and outcomes of infants transferred from NICU after 44 weeks CGA over a 5 year period.

Methods Eligible infants transferred from NICU between 1/09/ 2010 - 31/08/2015 were identified from the Standardised Electronic Neonatal Database (SEND) and PICU databases. Information was extracted from the SEND discharge summaries and medical records regarding transition of care.

Results 26 infants were identified. A mean of 4 (1-6) infants were transitioned annually to PICU or the paediatric wards. Median birth gestation was $36+{ }^{2}\left(23+{ }^{6}-41+{ }^{1}\right)$ weeks, weight of $2400(515-4480)$ grams. Median transition age was $46+{ }^{1}$ $\left(44+{ }^{0}-52+{ }^{6}\right)$ weeks, weight of $3417(1890-6170)$ grams. 11 of the 26 infants identified were transferred from NICU to PICU. Median length of stay (LOS) on PICU was 28 (1-241) days, mean 47 days. PICU occupancy per annum was 517 days, $13 \%$ of the annual bed occupancy (3754 days). 22 infants were transferred to the general paediatric wards, including some of whom were initially on PICU. Median LOS on the general paediatric ward was 11 (1-145) days, total of 591 days annually. No child died before discharge from hospital.

Conclusion This small group of complex infants constitute a high workload for both PICU and the general paediatric wards. The majority of infants remaining in hospital post 44 weeks CGA had undergone surgery during the neonatal period. The knowledge obtained from this study will help in planning the timely transition of these infants to paediatric services. This may help prevent local capacity issues to paediatric services by neonates awaiting repatriation around the region and familial separation. 